Regards sur l'économie allemande

Bulletin économique du CIRAC

$87 \mid 2008$

Varia

\title{
Des entreprises allemandes bien positionnées en Chine
}

Solène Hazouard

\section{OpenEdition}

\section{Journals}

Édition électronique

URL : http://journals.openedition.org/rea/969

DOI : $10.4000 /$ rea.969

ISBN : 978-2-8218-0870-6

ISSN : 1965-0787

Éditeur

CIRAC

Édition imprimée

Date de publication : 1 juillet 2008

Pagination : 5-12

ISSN : 1156-8992

\section{Référence électronique}

Solène Hazouard, «Des entreprises allemandes bien positionnées en Chine », Regards sur l'économie allemande [En ligne], 87 | juillet 2008, document 1, mis en ligne le 01 juillet 2010, consulté le 01 mai 2019. URL : http://journals.openedition.org/rea/969; DOI : 10.4000/rea.969 


\section{Des entreprises allemandes bien positionnées en Chine}

\section{Solène Hazouard}

Contre toute attente, la Chine n'a pas surclassé l'Allemagne qui confirme pour la sixième fois en 2008 son titre de championne du monde à l'export. Selon l'Agence fédérale pour la promotion du commerce extérieur (bfai), c'est l'euro fort qui lui a permis de se maintenir devant la Chine qui, avec 1220 milliards \$ d'exportations (source: OMC), a ravi en 2007 la seconde place aux Etats-Unis. Car la Chine a su rapidement se hisser au rang de géant commercial : ne seraitce qu'à l'échelle sino-allemande, le volume des échanges a quasi triplé depuis l'an 2000. Dans le même temps, elle est passée du dixième au troisième rang des pays fournisseurs de l'Allemagne, talonnant la France et les Pays-Bas. Egalement onzième acheteur mondial de produits « made in Germany », la Chine est aujourd'hui le principal partenaire commercial de l'Allemagne en Asie.

Cet essor des relations économiques entre les deux pays a pour corollaire un ancrage plus profond des entreprises allemandes sur le sol chinois, motivé en premier lieu par le potentiel de croissance de son marché intérieur. La volonté d'assurer la pérennité des réseaux de partenariat entre grands groupes et soustraitants à l'échelle locale, la faiblesse des coûts de production et d'approvisionnement ainsi que la possibilité, depuis la Chine, de s'ouvrir à d'autres marchés asiatiques, sont autant de facteurs additionnels d'implantation révélés par une étude récente de la Chambre de commerce allemande en Chine.

Depuis 2004, l'Union européenne est le premier partenaire économique de l'Empire du Milieu, représentant $15 \%$ de l'ensemble du commerce extérieur chinois. Les échanges Chine-UE tiennent une place essentielle dans les relations économiques mondiales : le Centre de recherche du Congrès américain estime qu'en 2007 , le volume du commerce bilatéral devrait avoir dépassé le niveau des 300 milliards $€$ (contre près de 280 milliards $€$ pour les échanges sino-américains). Les relations sino-européennes sont tirées par l'Allemagne qui, en 2006, réalisait déjà à elle seule près d'un tiers du commerce extérieur de l'UE avec la Chine. Avec 84,6 milliards $€$ de biens échangés l'an passé (+10\% par rapport à 2006), l'Allemagne est aujourd'hui le sixième partenaire commercial de la Chine. En retour, la Chine occupe le huitième rang dans les échanges économiques de l'Allemagne ainsi que le second rang dans ceux de l'UE, après les Etats-Unis.

Les dix principaux partenaires commerciaux de l'Allemagne (2007)

\begin{tabular}{|lcc|}
\hline & Volume d'échanges $(e n$ mrd $€$ ) & Part dans les échanges commerciaux \\
France & 158,8 & $9,1 \%$ \\
Pays-Bas & 126,7 & $7,3 \%$ \\
Etats-Unis & 119,0 & $6,8 \%$ \\
Royaume-Uni & 114,4 & $6,6 \%$ \\
Italie & 109,5 & $6,3 \%$ \\
Belgique & 90,2 & $5,2 \%$ \\
Autriche & 85,5 & $4,9 \%$ \\
Chine & $\mathbf{8 4 , 6}$ & $\mathbf{4 , 9} \%$ \\
Espagne & 69,3 & $4,0 \%$ \\
Suisse & 66,1 & $3,8 \%$ \\
Total & $\mathbf{1 7 4 1 , 6}$ & $\mathbf{1 0 0} \%$ \\
\hline
\end{tabular}

Source des données : Zusammenfassende Übersichten für den Außenhandel - Vorläufige Ergebnisse - 2007, Statistisches Bundesamt, Wiesbaden, 2008 (www.destatis.de) ; calculs de l'auteur. 
Déficit commercial avec la Chine pour l'Allemagne comme pour l'UE

La Chine exporte un volume croissant de produits à haute valeur technologique... ...tandis que la spécialisation sectorielle allemande s'intensifie
Bien que la balance commerciale allemande ait affiché un excédent mondial record de $+196,5$ milliards $€$ en 2007 , le déficit avec la Chine s'est nettement creusé depuis 2004 pour atteindre 24,7 milliards $€$. II reste toutefois proportionnellement inférieur à celui de l'UE, qui s'élevait à plus de 128 milliards € en 2006 (soit une hausse de $57 \%$ par rapport à 2003), et qui, selon les estimations d'Eurostat, devrait atteindre pour l'ensemble de l'année 2007 près de 160 milliards $€$. Sur la seule période de janvier à mai 2007, le déficit européen a augmenté de $53 \%$, en raison notamment d'une progression de $138 \%$ des exportations de produits sidérurgiques chinois vers l'Union européenne. L'importance croissante du déficit commercial constitue un défi stratégique dès lors que les économies estasiatiques ont centralisé leurs productions sur le territoire chinois, qui assume actuellement une fonction d'assembleur final.

En 2007, l'Allemagne a importé pour 54,6 milliards $€$ de biens en provenance de la Chine. D'une valeur de 48,8 milliards $€$ en 2006 , les importations allemandes comptaient pour un quart de celles de l'UE dont la Chine est devenue en 2006 la première source d'importations avec près de 192 milliards $€$ ou 14,4 \% du total des importations. Les produits à haute valeur technologique tiennent aujourd'hui une place croissante dans les achats allemands : la bureautique et les équipements informatiques sont représentés à hauteur de 18,6\%, contre 10,4\% en 2000 ; le secteur de l'électronique et des télécommunications a vu sa part croître de 4 points pour atteindre 16,5\%. A l'inverse, celle des biens à plus faible valeur ajoutée comme les meubles, jouets et bijoux est en léger recul, passant de $11,1 \%$ à $9,9 \%$ en l'espace de sept ans, de même que celle de l'habillement $(9,7 \%$ contre $11,5 \%)$. Les chambres de commerce et d'industrie allemandes s'attendent à ce que les importations allemandes originaires de Chine atteignent 72 milliards $€$ en 2008 , soit une hausse de près de $80 \%$ par rapport à 2005, ce qui placerait la Chine au deuxième rang des pays fournisseurs de l'Allemagne.

Chine : évolution des importations et exportations allemandes par secteurs (2000/2007)

\begin{tabular}{|lrr|}
\hline Exportations & Part en 2000 & Part en 2007 \\
Construction mécanique & $24,0 \%$ & $29,0 \%$ \\
Automobile & $6,0 \%$ & $16,0 \%$ \\
Electrotechnique & $6,0 \%$ & $10,0 \%$ \\
Produits chimiques & $8,0 \%$ & $9,0 \%$ \\
Fer et acier & $5,0 \%$ & $8,0 \%$ \\
Mesure et asservissement & $4,0 \%$ & $6,0 \%$ \\
Autres & $47,0 \%$ & $22,0 \%$ \\
Total (en mrd €) & $\mathbf{9 , 5}$ & $\mathbf{2 9 , 9}$ \\
\hline Importations & & \\
Bureautique/Informatique & $10,0 \%$ & $18,0 \%$ \\
Electronique/Télécommunications & $12,0 \%$ & $16,0 \%$ \\
Textile/Habillement & $16,0 \%$ & $13,0 \%$ \\
Meubles/Jouets/Bijoux & $11,0 \%$ & $10,0 \%$ \\
Electrotechnique & $10,0 \%$ & $7,0 \%$ \\
Construction mécanique & $6,0 \%$ & $7,0 \%$ \\
Autres & $35,0 \%$ & $29,0 \%$ \\
Total (en mrd €) & $\mathbf{1 8 , 6}$ & $\mathbf{5 4 , 6}$ \\
\hline
\end{tabular}

Source des données : Handelsblatt, 21-04-2008 (Bundesbank, Statistisches Bundesamt).

En ce qui concerne les biens exportés de l'Allemagne vers la Chine, qui représentent à eux seuls non moins de $43 \%$ du total de l'UE (source: Eurostat), l'Allemagne se retrouve bien positionnée avec ses secteurs-phares traditionnels: la construction mécanique tient le haut du pavé avec $29 \%$ en 2007 (contre $24 \%$ en 2000). C'est précisément dans les secteurs de l'automobile, de l'électrotechnique, de la construction mécanique et de l'industrie chimique/pharmaceutique que l'Allemagne dispose des meilleurs avantages comparatifs et réalise $60 \%$ de ses exportations à l'échelle mondiale (source: Prognos). La demande chinoise en équipements mécaniques correspond aux besoins d'une économie en voie d'industrialisation telle que la Chine, aujourd'hui l'un des principaux pays importateurs du monde dans ce domaine, dont le propre volume d'exportations de 
machines a été par ailleurs multiplié par deux entre 2004 et 2006 . Autres grands secteurs traditionnels à l'export : l'automobile, qui compte actuellement pour $16 \%$ des biens vendus à la Chine, l'électrotechnique (10\%) et les produits chimiques (9\%), contre respectivement $6 \%, 6 \%$ et $8 \%$ en 2000 . L'offre allemande satisfait ainsi pleinement la demande chinoise.

Malgré le boom récent des échanges, la Chine n'absorbe toujours que $3 \%$ des exportations et $1 \%$ des investissements allemands : les possibilités d'évolution restent considérables. Depuis 1999, l'Allemagne est le principal investisseur européen en Chine en volume annuel d'IDE et occupe la neuvième place mondiale, largement derrière Hong-Kong, les Etats-Unis et Taïwan. En 2006, les entreprises allemandes y ont investi 1,98 milliards $\$$, pour une valeur cumulée de 14 milliards $\$$.

Les principaux secteurs d'investissement sont ceux de la construction mécanique, de l'automobile et de l'industrie pharmaceutique qui, lors de la première phase d'ouverture économique de la Chine dans les années 1980, envisageaient avant tout l'Empire du Milieu comme un site de production à bas coût. En retour, cette concentration des investissements dans quelques grands secteurs industriels répond parfaitement à une demande déterminée par le gouvernement chinois dans le cadre du processus de développement économique du pays. Pour ce qui est de la construction mécanique, l'industrie chinoise, devenue le quatrième fournisseur mondial de machines après les Etats-Unis, le Japon et l'Allemagne, émet toujours un fort besoin d'équipements en vue de sa modernisation.

Les investissements relatifs au secteur de l'automobile sont quant à eux motivés par la volonté caractéristique d'une industrie de biens de consommation de se trouver au plus près de la demande, incarnée par une classe moyenne émergente d'environ 200 millions de personnes. Le potentiel est impressionnant : on s'attend à ce que 140 millions d'automobiles soient en circulation en Chine en 2020, contre 29 millions en 2006. Et Volkswagen, qui possède à Shanghai l'usine automobile allemande la plus ancienne, la plus célèbre et la plus prospère en Chine, semble particulièrement bien tirer son épingle du jeu en termes de positionnement : grâce aux ses deux joint ventures avec les firmes chinoises SAIC et FAW, le groupe possédait en 2005 la plus grande part de marché sur le segment des voitures particulières, devant General Motors, Hyundai/Kia, Honda, Suzuki et Mazda. Toutefois, les constructeurs DaimlerChrysler, BMW et Audi, filiale de Volkswagen, sont aussi très actifs sur le territoire chinois.

Dans le domaine de la chimie, la Chine est devenue le troisième marché ainsi que le troisième producteur mondial, derrière les Etats-Unis et le Japon. Les groupes BASF, Bayer, Hoechst, Agfa, Henkel ou encore Linde (gaz industriel) ont opté depuis de nombreuses années pour un investissement stratégique. BASF est le premier investisseur allemand en Chine, la construction de son usine de Nanjing constituant par ailleurs l'un des principaux investissements étrangers sur le territoire chinois. Outre l'industrie, le secteur des banques et assurances (Allianz), les maisons d'édition et les transporteurs allemands ainsi que de nombreux sous-traitants et PME ont choisi d'établir des filiales en Chine, reconnaissant ainsi le facteur déterminant du marché dans leur positionnement concurrentiel futur.

\section{La stratégie d'implantation des entreprises allemandes}

La répartition des entreprises allemandes en Chine indique une forte concentration autour des grandes agglomérations côtières orientales, structurée en trois grandes régions industrielles. La zone de Pékin, Tianjin, du Liaoning et du Shandong se consacre particulièrement aux industries automobile, chimique, des matières premières et de l'acier. Elle abrite par ailleurs un nombre croissant d'entreprises high-tech. Au sein de la région, les villes de Pékin et Tianjin ont attiré avant tout des fabricants de software et de téléphones mobiles. La zone du delta
Investissements en Chine : un potentiel à développer

L'offre allemande rencontre une demande chinoise définie par le politique

L'automobile sert la demande de la classe moyenne émergente

Chimie, finance et logistique : un positionnement stratégique

Les entreprises allemandes sont regroupées dans l'est industriel de la Chine 
du Yang-Tsé, regroupant les villes de Shanghai et les provinces du Jiangsu, du Zhejiang et de l'Anhui, est quant à elle plutôt spécialisée dans les semi-conducteurs et équipements informatiques. C'est ici que sont rassemblées, à proximité de Shanghai, la moitié des 4000 entreprises allemandes implantées en Chine. Enfin, le delta du Zhu Jiang (ou Rivière des Perles), composé des provinces du Fujian et du Guangdong, s'oriente vers la production d'appareils électroménagers, de téléphonie et d'équipements informatiques.

\section{Répartition géographique des entreprises allemandes sur le territoire chinois}

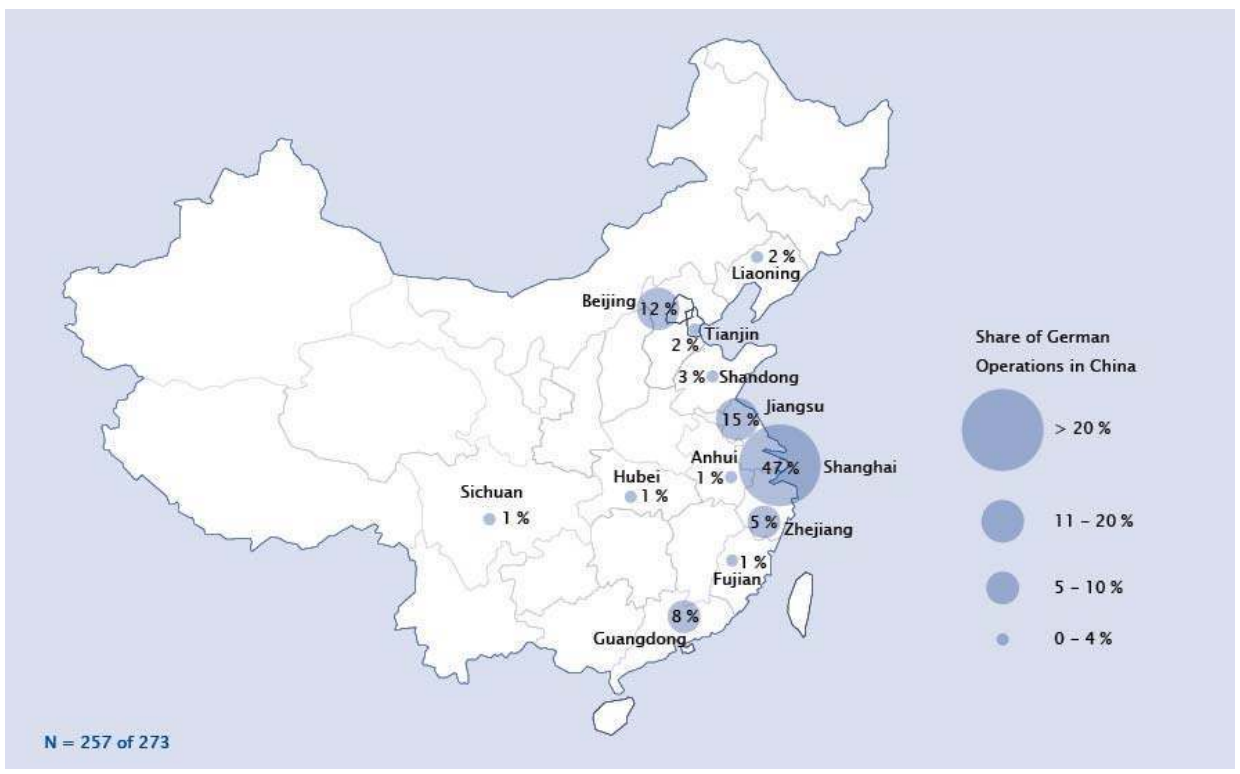

Source : Chamber of Commerce in China and EAC-Euro Asia Consulting PartG, Munich/ Shanghai/ Mumbai.

\section{Taicang, un 'village allemand'}

L'exemple de la ville de Taicang, située à quelques kilomètres de Shanghai, est révélateur de la haute densité d'entreprises allemandes au sein d'une zone géographique propice à leur implantation. En avril 2008, 109 d'entre elles, la plupart issues du Land de Bade-Wurtemberg, avaient fait le choix de ce site. Ce phénomène a vu le jour au début des années 1990, avec l'implantation de l'entreprise de ressorts automobiles Kern-Liebers, suivie rapidement par d'autres sociétés du Mittelstand comme Stihl (1995), Fischer (1996), ou encore Trumpf (2001) qui ont profité de la reconnaissance, au sein de ce 'village' de 450000 habitants, de la culture des affaires outre-Rhin. En effet, les valeurs soi-disant typiquement allemandes d'honnêteté, de ponctualité, d'équité et de sincérité semblent hautement appréciées en Chine (Kausch, 2007). Avec la hausse des coûts dans les grandes villes, Taicang se révèle un choix judicieux : le prix de location d'une usine y est trois fois moins élevé qu'à Shanghai. Reste à s'atteler à un problème capital : le manque de main-d'œuvre qualifiée. Pour y remédier, les entreprises ont mis sur pied il y a sept ans un centre de formation allemand pour mécaniciens outilleurs, qui propose 26 places d'apprentissage chaque année. L'examen se déroule selon les modalités des chambres de commerce et d'industrie allemandes, les apprentis se voyant attribuer le certificat allemand de compétence professionnelle.

Evolution de la structure des entreprises implantées en Chine
Selon l'étude German Business Expansion in China : 2008-2010, menée conjointement par la Chambre de commerce allemande en Chine et l'Euro Asia Consulting PartG (EAC) auprès de 273 entreprises allemandes, le principal facteur d'investissement réside dans le potentiel d'un marché de 1,3 milliard d'habitants, qui reste à relativiser cependant en termes de pouvoir d'achat : la majeure partie de la population n'est pas encore en mesure de s'offrir les biens de consommation proposés par les entreprises occidentales. En revanche, dans les domaines électrotechniques liés aux infrastructures et à l'énergie, il existe bel et bien une demande visant à désenclaver les sites économiques du pays. En raison de la politique d'ouverture du gouvernement chinois envers les investissements étrangers dans les secteurs de la distribution, de la logistique, de la finance et des assurances, les entreprises allemandes en Chine, d'implantation relativement récente (la plupart d'entre elles s'y sont établies après l'an 2000), s'orientent de plus en plus vers les services ou la distribution, bien que $68 \%$ aient encore une activité de production. De la même manière que pour les échanges commerciaux, l'implantation d'entreprises dénote une forte spécialisation sectorielle allemande. 
Les entreprises et bureaux de représentation allemands en Chine, par secteur et type d'activités

\begin{tabular}{|lccc|}
\hline Secteur d'activité & Part d'entreprises & Production & Services/Distribution \\
Construction mécanique & $29 \%$ & $87 \%$ & $13 \%$ \\
Automobile & $16 \%$ & $79 \%$ & $21 \%$ \\
Biens de consommation & $11 \%$ & $67 \%$ & $33 \%$ \\
Industrie chimique/pharmaceutique & $10 \%$ & $78 \%$ & $22 \%$ \\
Construction & $6 \%$ & $64 \%$ & $36 \%$ \\
Environnement/Energie & $6 \%$ & $63 \%$ & $37 \%$ \\
IT/Télécommunications & $3 \%$ & $44 \%$ & $56 \%$ \\
Finance & $3 \%$ & $100 \%$ & $0 \%$ \\
Autres & $15 \%$ & - & - \\
Total & $100 \%$ & - & - \\
\hline
\end{tabular}

Source des données : Chamber of Commerce in China and EAC- Euro Asia Consulting PartG, Munich/ Shanghai/ Mumbai.

\section{Siemens, un 'pionnier' des échanges industriels sino-allemands}

L'intérêt des entreprises allemandes pour la Chine n'est pas nouveau : les 'pionniers' se sont tournés vers l'Empire du Milieu au début de l'ère industrielle, dans la première phase de 'mondialisation'. Parmi eux, de grands noms industriels allemands tels ThyssenKrupp, Bosch ou encore Siemens, dont l'histoire en Chine est particulièrement révélatrice de la nature des échanges commerciaux sino-allemands jusque dans les années 1970 (Reisach, 2003). Avec plus de 60 sociétés dans de nombreux domaines ayant trait aux infrastructures, le groupe Siemens en Chine, dont l'appellation «Xi Men Zi » signifie « le maître entré par la porte de l'occident », fait partie des entreprises allemandes les mieux réputées au sein de l'Empire du Milieu.

Dès 1872, après seulement vingt-cinq années d'existence, l'entreprise a commencé à livrer des télégraphes à aiguilles sur le territoire chinois. Dès 1904, avant même la proclamation de la République de Chine par Sun Yat-sen, une première filiale est créée à Shanghai. Après avoir surmonté les difficultés financières inhérentes à l'effort de reconstruction des années 1920, Siemens connaît un essor prodigieux en Chine : en 1937, peu avant la guerre sino-japonaise, la filiale de Shanghai, dont les trois quarts des collaborateurs sont chinois, constitue la première implantation de Siemens en dehors de l'Europe, contribuant à elle seule à hauteur de $21 \%$ au chiffre d'affaires réalisé par les onze filiales de la zone hors Europe. A l'issue de la Seconde Guerre mondiale, la République populaire de Chine est fondée en 1949 : la politique d'autarcie menée alors par Mao Zedong gèle les relations économiques extérieures. Ainsi dans les deux décennies qui vont suivre, nombre d'entreprises étrangères ne pourront atteindre le marché de la République populaire de Chine que de manière indirecte, par exemple via la colonie britannique de Hong Kong dans le cas de Siemens.

Face à des difficultés économiques croissantes, la Chine amorce un dialogue diplomatique à l'international dès 1972. Mais l'étape décisive dans l'ouverture économique ne sera franchie que six ans plus tard: en accord avec les réformes initiées par Deng Xiaoping, la politique sera désormais axée sur l'opposition entre la productivité insuffisante de l'économie nationale et les besoins matériels de la population (Reisach/Meier, 2008). A la réforme agricole suit en 1984 une réforme profonde de l'industrie. On attend alors des entreprises internationales qu'elles injectent du capital et du savoir au sein de joint ventures, avec un objectif précis : proposer des produits de rang mondial du point de vue de la qualité et du design, qui seront destinés à être exportés en grande partie sur des marchés tiers. En effet, la Chine doit alors accroître considérablement son stock de devises pour financer ses importations de bien intermédiaires de type acier, engrais, matières plastiques et fibres synthétiques mais aussi d'automobiles, avions et produits industriels à forte valeur technologique. Dans le même temps, cette ouverture vers l'occident est stimulée par la volonté d'améliorer la formation des collaborateurs chinois et de bénéficier de transferts de technologies.

C'est dans ce contexte que nombre de grandes entreprises allemandes vont installer des sites de production en Chine, à l'instar de ThyssenKrupp ou encore Volkswagen, qui fonde en 1985 à Shanghai la première joint venture internationale dans le secteur de l'automobile. La même année, Siemens signe un «Mémorandum pour la coopération entre la République populaire de Chine et Siemens AG dans les domaines de la construction mécanique, de l'industrie électrique et électronique " destiné à moderniser ces branches industrielles en Chine et qui garantira à Siemens d'importantes commandes les années suivantes. En 1990, l'entreprise franchit le pas de la production en créant la Beijing International Switching System Corporation Ltd. (BISC), dans le secteur des télécoms. Au fil des années, de nombreux sous-traitants, galvanisés par une certaine atmosphère de 'conquête de l'Est', décideront de suivre les grands groupes en Chine de sorte que les PME représentent désormais non moins de $40 \%$ des entreprises implantées dans l'Empire du Milieu.

Dernière grande étape chinoise de l'insertion de la Chine dans le nouveau partage mondial du travail, son adhésion à l'OMC en décembre 2001, avec pour corollaire la disparition progressive des tarifs douaniers, la fin des quotas d'importation et licences en 2006 et la possibilité, pour les entreprises à capitaux étrangers, d'importer et d'exporter des biens dans leur propre secteur d'activité. Dans ce contexte, un accord sino-allemand destiné à promouvoir et protéger des investissements bilatéraux a été conclu en 2003, pour entrer en vigueur en 2005. Cependant certaines attitudes protectionnistes ont tendance à resurgir : la Chine a refusé de signer l'accord des membres de l'OMC concernant l'ouverture des marchés publics aux entreprises étrangères, privilégiant ainsi les entreprises domestiques lors de l'attribution de grands projets d'infrastructures nationaux.

Malgré cela Siemens tire toujours son épingle du jeu sur le marché chinois : d'ici 2010, l'entreprise doit livrer 60 trains à grande vitesse Velaro (une version plus large et plus rapide de l'ICE 3 qui relie notamment Cologne à Francfort, pouvant atteindre une vitesse de $350 \mathrm{~km} / \mathrm{h}$ ), dont les cinq premiers modèles devront assurer dès cet été la liaison entre Pékin et la ville côtière de Tianjin. Ces trains, qui rapporteront la somme de 670 millions $€$ au groupe, seront produits en Chine au sein d'une joint venture. Par ailleurs, Siemens a reçu l'an dernier une commande de 500 locomotives de fret d'une valeur de 334 millions $€$, et pour 30 millions $€$, le groupe équipera la ligne de métro permettant d'accéder à la zone olympique de Pékin. Siemens espère désormais un allongement de la ligne du Transrapid jusqu'à Shanghai, en vue de livrer les équipements électroniques relatifs au projet. 
De la joint venture à la wholly foreign-owned enterprise

La fin des barrières tarifaires et les nouvelles préoccupations des dirigeants allemands

30 ans de coopération technologique bilatérale

Les grands instituts allemands travaillent en partenariat avec leurs homologues chinois
Parmi les implantations allemandes en Chine, $73 \%$ sont des entreprises et $27 \%$ des bureaux de représentation dont la part a décliné ces dernières années, à l'exception de la ville de Pékin où le lobbying auprès du gouvernement chinois est très intense. Parmi ces entreprises, on retrouve $71 \%$ de sociétés à capitaux exclusivement étrangers (wholly foreign-owned enterprises, WFOE) et $29 \%$ de sociétés à capitaux sino-étrangers (joint ventures). Par le passé, la création d'une joint venture était le seul moyen pour une entreprise étrangère de s'implanter en Chine, la création d'entreprises à capitaux exclusivement étrangers étant limitée, voire prohibée dans de nombreux secteurs industriels. Avec la levée progressive de ces interdictions, la part des WFOE a connu ces dix dernières années une hausse continue. Par ailleurs, la part des PME augmente, attirées par les grands groupes qui conservent ainsi leur réseau de fournisseurs à l'échelle locale.

Bien que la plupart des entreprises atteignent voire dépassent leurs objectifs en Chine (il faut compter en moyenne quatre ans pour qu'une entreprise de production réalise des profits ; pour une entreprise de services, ce délai est réduit à deux ans), elles doivent faire face à de nombreux obstacles. Certes, les barrières tarifaires ont progressivement disparu et les conditions cadres relatives aux investissements étrangers ont été améliorées en vue d'attirer notamment les PME. Toutefois il ne faut pas oublier que «la Chine reste encore très éloignée d'une économie de marché de type occidental. Les notions d'Etat de droit, de liberté et de démocratie sont et demeurent des valeurs occidentales auxquelles la Chine n'aspire pas au même degré » (Reisach/Tauber/Yuan, 2007). Ainsi, les investisseurs attendent une meilleure sécurité juridique (les lois adoptées ne sont pas systématiquement appliquées), une liberté de contracter étendue, ainsi qu'un accès aux marchés publics comparable à celui réservé aux entreprises chinoises. Des facteurs additionnels de blocage résident dans les problèmes de corruption et le manque de personnel qualifié. Par ailleurs, l'adoption d'une nouvelle loi relative à l'impôt sur les sociétés selon laquelle les aides seront désormais définies suivant la branche d'activité (avec une priorité accordée aux entreprises high-tech et de l'environnement), a mis brusquement fin aux privilèges fiscaux dont disposaient jusqu'à présent les entreprises étrangères. Mais ce qui préoccupe encore et surtout les chefs d'entreprise allemands qui ont choisi de s'implanter sur le marché le plus concurrentiel au monde relève des questions de protection des droits de propriété intellectuelle.

Malgré le risque bien réel des transferts de technologies illégaux, l'Allemagne a misé de manière relativement précoce sur la coopération scientifique et technologique avec la Chine, avec la signature en 1978 d'un accord gouvernemental bilatéral. L'année suivante, la Chine et l'Allemagne décident de mettre en place un échange sur le système normatif allemand (DIN) : les voyages diplomatiques de spécialistes techniques et représentants économiques se multiplient, de sorte qu'au fil du temps, de nombreux standards sont adoptés en Chine sur le modèle allemand. Un nouveau mode de coopération sur projets est mis en place, relayé ensuite par une coopération institutionnelle qui aboutit à la création de centres de recherche binationaux en 2004 et 2005 . Le ministère fédéral de la Formation et de la Recherche travaille ainsi en partenariat avec le ministère chinois de la Science et de la Technologie ainsi que le ministère chinois de l'Education.

Outre la collaboration scientifique intergouvernementale, les organismes de recherche allemands extra-universitaires de droit privé à but non lucratif ont instauré une coopération directe avec leurs partenaires chinois : ainsi le Centre SinoAllemand pour la Promotion de la Recherche (Deutsch-Chinesisches Zentrum für Wissenschaftsförderung), géré conjointement par la Fondation allemande pour la recherche (Deutsche Forschungsgemeinschaft, DFG) et le National Natural Science Foundation of China a vu le jour à Pékin. Les Instituts Fraunhofer ont ouvert par ailleurs deux centres de recherche en partenariat avec le High Technology Research and Development Center: le Sino-German Joint Software Institute (JSI) à Pékin et le Sino-German Mobile Communication Institute (MCI) à Berlin. L'Institut Max-Planck a quant à lui ouvert en 2005 un centre de Computational 
Biology à Shanghai, en collaboration avec l'Académie chinoise des sciences. Enfin, la Communauté Leibniz travaille depuis quatre ans à la mise en place d'un réseau reliant entre eux ses propres instituts, des instituts de recherche chinois et des partenaires industriels, afin de pouvoir isoler, caractériser et expérimenter des substances biologiques actives issues des plantes de la médecine traditionnelle chinoise. Ainsi, la coopération sur projets entre chercheurs allemands et chinois s'est développée de manière continue. Elle concerne aussi bien le domaine high-tech que la recherche environnementale et fondamentale.

La coopération technologique reste déterminante dans le processus de modernisation de l'économie chinoise. Dans les années à venir, les efforts vont converger vers le développement des infrastructures à l'échelle nationale. Cela implique un approvisionnement en énergies efficientes et non polluantes, des moyens de transports performants, un accès étendu aux technologies de l'information et de la communication ainsi que la modernisation du tissu industriel. Mais le plus grand défi reste celui de l'urbanisation et de la construction future de bâtiments 'écologiques' à faible consommation d'énergie. Dans ce contexte, les entreprises allemandes qui exportent, produisent, forment, créent des emplois hautement qualifiés et collaborent en termes de R\&D ont jeté les bases d'une participation active aux projets d'expansion économique chinoise.

La représentation institutionnelle des entreprises allemandes passe par la Chambre de commerce allemande en Chine qui possède des bureaux de délégués à Pékin, Shanghai et Canton. Cette institution reflète l'engagement des grands industriels dans la coopération économique sino-allemande : elle est dirigée par le président de Siemens en Chine, Richard Hausmann, qui compte parmi ses collaborateurs des représentants de ThyssenKrupp, Bayer, Siemens ou encore Volkswagen. Les activités est-asiatiques des fédérations de l'industrie allemande (BDI), des chambres de commerce et d'industrie (DIHK), des banques $(\mathrm{BdB})$ et du commerce extérieur et de gros (BGA) ainsi que les travaux de l'association spécialiste de l'Asie qu'est l'Ostasiatischer Verein (voir dans ce numéro l'entretien avec Monika Stärk, membre de son bureau exécutif) sont par ailleurs coordonnées d'Allemagne depuis 1993 par le Comité Asie-Pacifique de l'Economie Allemande (Asien-Pazifisches-Ausschuss der Deutschen Wirtschaft, APA), avec à sa tête l'actuel président de BASF, Jürgen Hambrecht. Sur le sol chinois, les German Centres de Pékin et de Shanghai, issus de la coopération notamment entre banques publiques, fédérations professionnelles et ministères, sont des lieux de contact mettant à disposition des entreprises des locaux commerciaux et des salles de conférence.

A l'échelle gouvernementale (outre l'action des gouvernements des Länder qui mènent leur propre politique), l'Agence fédérale pour la promotion du commerce extérieur (Bundesagentur für Außenwirtschaftsförderung, bfai) du ministère fédéral de l'Economie fournit des informations détaillées sur les marchés, recueillies sur place par un réseau mondial de correspondants qui travaille en collaboration avec les ambassades et les $\mathrm{CCl}$ à l'étranger (voir REA 70/05). Via le portail IXPOS, le ministère oriente les entreprises exportatrices dans la recherche de clients et de partenaires commerciaux. L'institution publique Euler-Hermes-Kredit AG distribue quant à elle des crédits à l'exportation : 1,3 milliard $€$ ont été ainsi assurés en 2006 dans les échanges avec la Chine. Ce dispositif profite en grande partie aux grands groupes, $1 \%$ seulement des PME allemandes recourant aux garanties Hermes (voir REA 83/07).

LES ENTREPRISES ALLEMANDES ONT SAISI TRÈS TÔT LES ENJEUX de la coopération économique avec la Chine qui, grâce à une croissance annuelle de 9,5\% depuis 1990, est devenue la quatrième puissance économique et troisième puissance commerciale du monde. Mais avec un PIB de 2000 \$ par tête, et des disparités sociales extrêmement marquées dans un contexte de surchauffe économique (moins de $5 \%$ des ménages disposent d'un revenu annuel supérieur à 5000 \$), l'Empire du milieu demeure un pays en voie de développement dont le modèle
Le défi de l'urbanisation et du développement des infrastructures

La coopération sino-allemande repose sur les chefs d'entreprise et les fédérations professionnelles

Les aides publiques au commerce extérieur sont méconnues des PME 
économique a atteint ses limites : l'aspect social et environnemental avait été relégué au second plan dans l'accès au statut 'd'atelier du monde'. Ainsi le onzième plan quinquennal (2006-2010), qui prévoit de doubler la performance économique du pays d'ici 2010, préconise le respect de l'environnement ainsi qu'une réduction drastique de la consommation énergétique. L'enjeu est double : il s'agit non seulement de rassurer l'opinion internationale et les investisseurs dans le cadre des JO de Pékin, récemment ternis par la question tibétaine et la résurgence de mesures protectionnistes sévères, puis de l'exposition universelle de Shanghai 2010, mais aussi et avant tout de réduire un niveau de pollution tellement élevé qu'il représente un frein à la croissance (la Chine abrite 16 des 20 villes les plus polluées au monde) et de pallier, via des solutions innovantes, la pénurie d'eau potable et le manque de ressources énergétiques. Dans les dix années à venir, à l'échelle mondiale, un grand bâtiment sur deux sera construit en Chine : dans ce contexte, le pays aura besoin de constructions respectant des normes d'isolation modernes, de systèmes d'évacuation des eaux usées ou de centrales électriques que les entreprises allemandes sont précisément en mesure de proposer. Cependant, ces dernières risquent de passer à côté de ce marché colossal : la qualité des produits " made in Germany » demeure en partie trop élevée au regard des normes en vigueur en Chine, ce qui pourrait jouer en leur défaveur en termes de compétitivité...

\section{Indications bibliographiques}

BOURGEOIS I., «La place de l"Allemagne dans l'économie mondiale », et BouRGEOIS I., LASSERRE R., «Les PME allemandes : acteurs de la mondialisation », Dossier : «Spécial globalisation », Regards sur l'Economie Allemande, n83/2007

External and intra-European Union trade, Eurostat, septembre 2007

GABEL M., «Chine, Inde, Brésil : les choix des entreprises allemandes », Regards sur l'Economie Allemande, n70/2005

«German Business Expansion in China : 2008-2010», BusinessForum China Special Issue, German Chamber of Commerce in China and EAC - Euro Asia Consulting PartG, Munich/ Shanghai/ Mumbai, 2008

Globalisierungsreport 2008, Prognos, juin 2008

KaUsch U., China Pioniere - Unternehmer berichten von ihren Erfolgen im Reich der Mitte, Francfort/Main, 2007

LISBONNE-DE VERGERON K., « La dimension stratégique des relations commerciales et monétaires Chine-Union européenne », Questions d'Europe, Fondation Robert Schuman, n96, avril 2008

Meier R., ReISACH U., Aufbruch im Land des Drachen - Arbeiten und Leben in China zwischen Konfuzianismus, Sozialismus und Globalisierung, Gernsbach, 2008

REISACH U., «Deutsch-chinesische Wirtschaftszusammenarbeit-das Beispiel Siemens », in SCHÜLLER M., (ed.), Strukturwandel in den deutsch-chinesischen Beziehungen - Analysen und Praxisberichte, Mitteilungen des Instituts für Asienkunde, $\mathrm{n}^{\circ} 370$, Hambourg, 2003

REISACH U., TAUBER T., YUAN X., China-Wirtschaftspartner zwischen Wunsch und Wirklichkeit - Ein Handbuch für Praktiker - 4., aktualisierte Auflage, Heidelberg, 2007

www.asien-pazifik-ausschuss.de ; www.auswaertiges-amt.de ; www.bfai.de ; www.china.ahk.de ; www.ihk-koeln.de ; www.ixpos.de. 